\title{
HYPERSPECTRAL IMAGE VISUALIZATION WITH A 3-D SELF-ORGANIZING MAP
}

\author{
Johannes Jordan, Elli Angelopoulou \\ Pattern Recognition Lab, University of Erlangen-Nuremberg, Germany \\ johannes.jordan@cs.fau.de, elli.angelopoulou@cs.fau.de
}

\begin{abstract}
False-color visualization is a powerful component of interactive hyperspectral image analysis. We propose a novel unsupervised technique for false coloring that is based on self-organizing map (SOM) dimensionality reduction. We first train a modified 3-dimensional SOM on the image data. Instead of a single answer, our SOM returns several answers to each data query. Then we employ a novel rank-based linear weighting to create a meaningful RGB representation of the query result. We analyze and compare our visualization results on publicly available remote sensing and laboratory image data. The obtained false coloring is superior to established principal component based false coloring while retaining computational efficiency.
\end{abstract}

Index Terms - Hyperspectral imaging, Self-organizing feature maps, Visualization, False Coloring

\section{INTRODUCTION}

In a hyperspectral image (HSI) we essentially observe high dimensional data points that share a spatial relation. Visualizing the data is important where a manual observer is needed for interpretation, or for evaluation of an automated interpretation. While many approaches concentrate on the data points without spatial context, some methods create a display that follows the spatial layout of the image. The most simple form is an intensity image that presents a single data channel. However, the tri-chromatic human color perception allows us to distinguish three channels per pixel. The process of creating a color image from several channels of data is called false coloring.

Three categories of false coloring are very common in HSI analysis. First, three bands from the image are selected and then mapped to $\mathrm{R}, \mathrm{G}, \mathrm{B}$ color channels. Second, dimensionality reduction on the image data as a whole is performed, leading to three bands that cover most information in the image and are mapped to R, G, B. Third, intermediate data values are computed through other means, e.g. classification of pixels. Then, a combination of classification results forms the false-color image. The scope of this paper is the second category.

Our work lies in the context of interactive HSI analysis systems [1]. We strive for an unsupervised false-coloring method that aids the user when exploring an image without prior knowledge. The method needs to be fast to compute to be helpful in an interactive setting. We present a novel approach that fulfills these properties. We employ the self-organizing map (SOM) [2], which is a wellunderstood tool for topology-preserving dimensionality reduction. The SOM is often used for visualization of multivariate data, however, typically in a way that identifies clusters in the underlying variable distribution, not taking the image layout into account. We show how we can obtain a high-quality false coloring based on a 3D SOM. We obtain a meaningful coloring directly from the data without clustering or incorporating prior knowledge. See Figure 1 for a remote sensing example. When compared to the established unsupervised PCA false

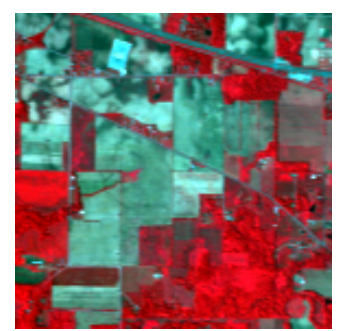

(a) Composite of three bands

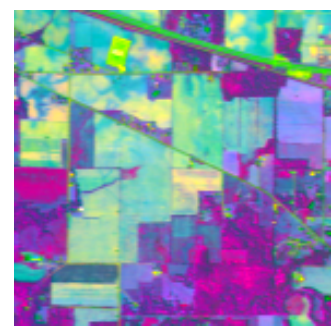

(c) PCA visualization

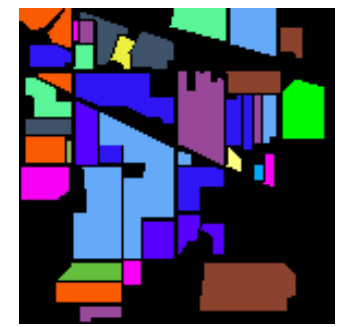

(b) ground truth labels

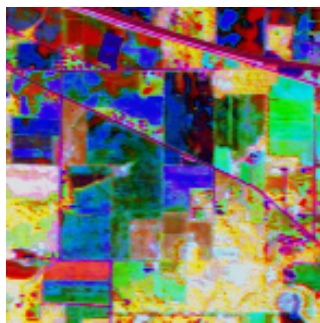

(d) 3D SOM visualization
Fig. 1. 220-band AVIRIS indian pines image. The proposed visualization is more feature-rich when compared to PCA false coloring.

coloring, our method produces superior results in multiple image capture modalities.

\section{UNSUPERVISED FALSE-COLOR VISUALIZATION}

Very prominent in false coloring are PCA-based methods. For PCA, eigenvalues are computed and sorted. The eigenvectors corresponding to the three largest eigenvalues form a linear transformation to three bands used as R, G, B after automatic white-balancing is performed [5]. Tyo et al. [3] propose a transform of the three principal components to HSV colorspace in an opponent-color fashion. Unfortunately, the method needs supervision for defining offsets to the second and third principal component. A notable derivation from PCA-based methods is described by Cui et al. [4] and also operates in HSV color space. To maximize spectral distance preservation, principle components form only $\mathrm{H}, \mathrm{S}$ components. The $\mathrm{V}$ component is then calculated by optimizing a $L_{2}$-based distance similarity objective. However, the informative value of the Euclidean distance for spectral similarity is arguable. The independent component analysis (ICA) [6] based visualization is a linear transformation similar to PCA that seeks mutually independent components in the data. However, it is not clear how to rank the significance of different channels [4].

Another approach to unsupervised false-color visualization lies in nonlinear dimensionality reduction techniques. Most prominent in this field is ISOMAP [7]. It seeks a manifold coordinate system 
that preserves geodesic distances in feature space. False-color images are created based on coordinates. Unfortunately, even after several improvements to algorithm complexity were made, ISOMAP still takes minutes to hours to compute [7, 4].

We compare the proposed method to PCA, as both methods share that they are unsupervised and fast to compute. While the PCA is a linear transformation, we base our method on the SOM, which is a nonlinear dimensionality reduction technique. However, a SOM can be trained on a HSI within seconds in contrast to other nonlinear methods [8].

\section{SELF-ORGANIZING MAPS}

The self-organizing map [2] was invented by Kohonen in 1982. The SOM can be used for reducing dimensionality of high-dimensional data points in a manner that preserves feature space topology [8]. A straightforward implementation and considerably short learning times make the SOM viable for several tasks in HSI analysis [9].

A SOM consists of an artificial neural network. The network is trained using unsupervised learning to convert the nonlinear statistical relationship between high-dimensional data into simpler geometric relationships. In other words, observed spectra are put into a topological relation that should best resemble their topology in the high-dimensional space.

We define the data of a HSI in the form $\mathbf{v}_{x, y} \in \mathbb{R}^{d}$, whereas $d$ is the number of spectral bands in the input image and $\mathbf{v}_{x, y}$ corresponds to an image pixel at position $x, y$. We omit subscript $x, y$ from here on for clearer presentation. The SOM consists of $n$ model vectors $\mathbf{m}_{i} \in \mathbb{R}^{d}$ (also called neurons). Let $d\left(\mathbf{v}, \mathbf{m}_{i}\right)$ denote a distance function for $\mathbf{v}$ and $\mathbf{m}_{i}$. The common choice for $d(\cdot)$ is the Euclidean distance, which is fine for learning the manifold. The best fit of $\mathbf{v}$ in the SOM, and therefore its best matching unit (BMU) $\mathbf{m}_{c}$, has the index

$$
c^{(\mathbf{v})}=\underset{i}{\operatorname{argmin}} d\left(\mathbf{v}, \mathbf{m}_{i}\right) .
$$

During training, $s$ vectors from the input image (or any other source) are randomly selected and fed into the SOM. After the BMU of input $\mathbf{v}(t), 1 \leq t \leq s$, is determined, a neighborhood function $h_{c, i}$ defines the influence of $\mathbf{v}(t)$ on the model vectors $\mathbf{m}_{i}$. As suggested in the literature [2],

$$
h_{c, i}(t)=\alpha(t) \cdot \exp \left(-\frac{\left\|\mathbf{r}^{(c)}-\mathbf{r}^{(i)}\right\|^{2}}{2 \sigma^{2}(t)}\right),
$$

where $\mathbf{r}^{(c)}, \mathbf{r}^{(i)}$ are location vectors of neurons $c$ and $i$, respectively. The location $\mathbf{r}^{(c)}$ of a neuron $\mathbf{m}_{c}$ in the SOM is determined by the SOM topology and is a bijective mapping of $c$. The learningrate factor $\alpha(t)$ is a user-adjustable parameter that is monotonically decreasing. The kernel width $\sigma(t)$ describes how far the influence of a sample vector reaches in the SOM topology and is also monotonically decreasing. While in the early training phase the SOM seeks a rough global ordering, in the later phase local regions are smoothed out.

\subsection{SOM for Visualization}

One major application of the SOM is in fact data visualization. Several well developed SOM-based techniques exist [10]. Typically, the data is visualized in the layout of the neuronal network, e.g. the U-matrix that encodes a neuron's distance to its neighbors and helps to manually identify clusters in the data. If the input data has a spatial context, a color-coding of a 1D or 2D SOM may be back-projected, e.g. for visualization of geo-referenced data on a map [11].
A mapping back to the spatial layout of a multispectral image was first proposed by Manduca [12]. However, they use a 1D SOM to produce a grayscale display. Most similar to our method is recent work that uses a 3D SOM for color-coded visualization of data in its original spatial layout. Gorricha and Lobo [11] train a SOM on geo-referenced data to color geographic elements. Fonville et al. [13] compare a 3D SOM with other data mappings for visualization of mass spectrometry imaging data.

Our work differs from these as we apply the method in the hyperspectral domain. To do so, we train considerably larger SOMs and propose a new method of processing several BMUs per look-up that leads to much higher quality RGB output.

\section{3D SOM VISUALIZATION}

Typically, SOM neurons are layed out in a 1D or 2D lattice, which is 2-connected, or 4-connected, respectively. A common deviation for the 2D SOM is a hexagon lattice. A 3D SOM is rarely used in the literature. When it is, a 6-connected lattice is constructed. Our SOM is cubic, such as the side length $n^{\prime}$ of the lattice in each dimension is $\sqrt[3]{n}$. With this topology, we obtain location vectors $\mathbf{r}^{(c)} \in \mathbb{Z}^{3}$ with $r_{i}^{(c)} \in\left[1, n^{\prime}\right]$. We exploit this fact to compute a HSI false coloring.

First, the SOM is trained on samples from the image. Then, the false-color values $r, g, b$ of a pixel $\mathbf{v}$ are obtained as

$$
\mathbf{r}_{x, y}=\frac{r_{1}^{\left(c^{(\mathbf{v})}\right)}}{n^{\prime}}, \quad \mathrm{g}_{x, y}=\frac{r_{2}^{\left(c^{(\mathbf{v})}\right)}}{n^{\prime}}, \quad \mathrm{b}_{x, y}=\frac{r_{3}^{\left(c^{(\mathbf{v})}\right)}}{n^{\prime}} .
$$

While this method can give a meaningful result on a per-pixel basis, the output suffers from quantization effects. A typical SOM is trained with $2^{8}$ neurons only, while we can display $2^{24}$ unique colors. To solve this problem and provide further accentuation of the data, we introduce a weighted interpolation scheme when querying the SOM. We first obtain a vector of BMU indices

$$
\mathbf{c}^{(\mathbf{v})}=\underset{\mathbf{i}}{\operatorname{argmin}} \sum_{j}^{C} d\left(\mathbf{v}, \mathbf{m}_{\mathbf{i}_{j}}\right),
$$

where $C$ is the number of desired BMUs, in analogy to [14]. Then, for each pixel $\mathbf{v}$, we calculate location $\mathbf{r}^{\prime}$ as

given

$$
\mathbf{r}^{\prime}=\sum_{j}^{C} w_{j} \cdot \mathbf{r}^{\left(c_{j}^{(\mathbf{v})}\right)}
$$

$$
\begin{aligned}
\forall w_{j}, j<C: & w_{j} & =2 w_{j+1} \\
\forall \mathbf{m}_{\mathbf{c}_{j}}, j<C: & \quad \sum_{j=1}^{C} w_{j} & =1 \\
& \left.=\mathbf{m}_{\mathbf{c}_{j}}\right) & <d\left(\mathbf{v}, \mathbf{m}_{\mathbf{c}_{j+1}}\right),
\end{aligned}
$$

which expresses that the BMUs are sorted according to distance to the query vector, and weighted by their rank, where the weight for rank $j$ is always twice as high as the weight for subsequent rank $j+1$. We finally obtain

$$
\mathrm{r}_{x, y}=\frac{r_{1}^{\prime}}{n^{\prime}}, \quad \mathrm{g}_{x, y}=\frac{r_{2}^{\prime}}{n^{\prime}}, \quad \mathrm{b}_{x, y}=\frac{r_{3}^{\prime}}{n^{\prime}} .
$$

Figure 2 shows an example multispectral image, (a), and false colorings obtained by a SOM with (b) $C=1$, resembling traditional SOM BMU lookup, (c) $C=10$ and $w_{j}=\frac{1}{C}, \forall j$, resembling an unweighted approach, and (d) $C=10, w_{j}$ determined as in Eq. 6. We observe that the traditional lookup of a single BMU depicted in 2(b) captures different materials, specular highlights and shadows well, but suffers from quantization effects. When adding more BMUs as in 2(c), a smooth display is obtained at the expense of significant details. Finally, our proposed weighting scheme displayed in 2(d) preserves most detail of all three methods in an artifact-free presentation. 


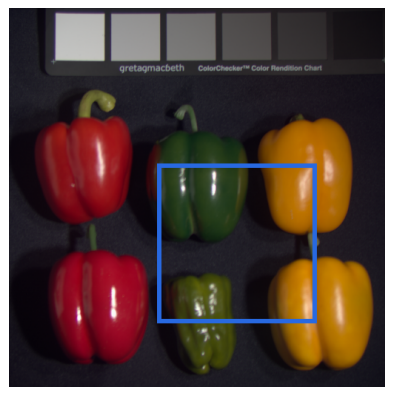

(a) Color matching functions

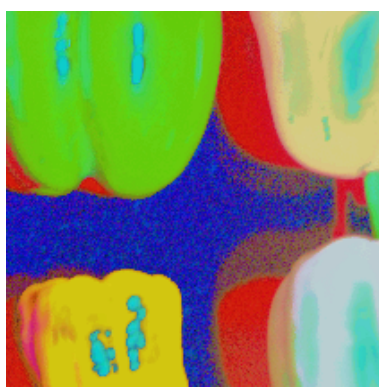

(c) SOM, 10 averaged BMUs

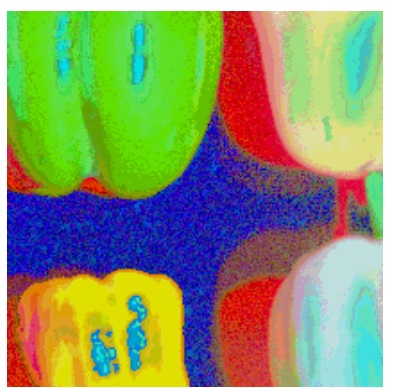

(b) SOM, single BMU

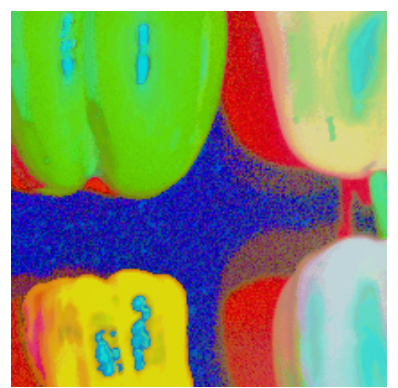

(d) SOM, 10 rank-weighted BMUs
Fig. 2. 31-band fake and real peppers image [15] and corresponding false-color visualizations. Results are zoomed-in for better visibility.

\section{EXPERIMENTAL RESULTS}

We examine our new method on a range of images from two capture modalities: Laboratory images taken with tunable filters on the ground and remote sensing images taken by AVIRIS and HYDICE sensors. The lab image shown in Figures 2,3 has a resolution of $508 \times 512$ pixels and covers the spectral range of $400 \mathrm{~nm}-700 \mathrm{~nm}$. For the lab images only, we operate on the spectral gradient space [16] to reduce the dominance of geometric effects, at the expense of an amplified noise level. The AVIRIS image shown in Figure 1 holds $145 \times 145$ pixels with the spectral range of $400 \mathrm{~nm}-2500 \mathrm{~nm}$, while the HYDICE image shown in Figure 4 holds $1280 \times 307$ pixels with the spectral range of $400 \mathrm{~nm}-2475 \mathrm{~nm}$.

Parameter setting. Each SOM is trained with $n=10^{3}, s=$ $100000, C=5$. The SOM quality can be assessed by comparing spectral distributions of SOM and image. We see a good match in Fig. 3(c,e). We found that a size $n$ of at least $6^{3}$ is needed to preserve all details in the image. Good choices for learning rate and kernel width of the SOM are easy to find [2] and we did not particularly fine-tune them as well as $s$. Parameter $C$ is not significant for $C \geq 5$.

Qualitative Assessment. Our goal for the image shown in Fig. 3 is a visualization that overcomes metamerism and therefore needs to significantly contrast with true color. We find that different materials of the peppers, three of which are plastic, are well separated by the SOM. You can discern plastic peppers from organic ones by looking at the coloring of their stems. Also, reflectance effects such as interreflections, specular highlights and shadowed regions are captured. The PCA visualization fails to distinguish the yellow peppers and the separation of the shadow on the backdrop is weak.

In the remote sensing image depicted in Fig. 4 the SOM provides a good separation of the classes grass, tree, roof, road, trail and water. We can also read more subtle details from the visualization, such as different roof types or different grass segments. For this image, the PCA also performs well. However, some structure is not as easy to distinguish, and some classes lack separation (e.g. water vs. road). In general we found that PCA often contrasts well a single class of pixels in the image (here a rooftop in white) at the expense of a good general contrast between other classes. The same effect is visible in Fig. 1. The PCA catches the steel tower in the upper right, but not more subtle differences between crop classes, effectively providing no benefit over the three-band composite in Fig. 1(a). While the SOM visualization illustrates the same effects as the other false-colorings, it also helps distinguish several of the classes shown in Fig. 1(b).

Quantitative Measures. A measure of the information content of R, G, B components, and therefore richness of the information, is per-component entropy. Entropy is increased by the proposed SOM in comparison to both alternatives shown in Fig. 2(b,c). Entropy is also consistently higher for SOM than for PCA and PCA has the problem that information content is uneven between components. Exemplary entropy values $E_{\mathrm{R}}, E_{\mathrm{G}}, E_{\mathrm{B}}$ are denoted below Fig. 4(a), 4(b).

On an Intel Core i7-2600 CPU, SOM training time in seconds is $10 s$ for the lab images, and $18 s$ for the remote sensing images due to higher spectral dimensionality. Spatial size is irrelevant. For SOMs of reduced size $n=6^{3}$, training times are $3 s$, and $5 s$, respectively.

Limitations. As the SOM sees a random set of pixels during training, the topology can significantly change in several runs. For an example see the different SOMs used in Figures 2, 3. Color difference between two image segments can change heavily, in consistence with this method not being distance preserving. However, our observation is that the SOM always captures the details we specifically look for when evaluating the results.

\section{CONCLUSIONS}

The SOM is known as a valuable tool for HSI analysis. It has the properties of being faster to compute than other nonlinear dimensionality reduction methods while retaining a more accurate representation of feature space topology than linear methods. We propose a SOM specialized for false-color visualization. Our SOM is 3-dimensional, holds a high number of neurons, and returns a linear combination of best matching units (BMUs) in the final data queries instead of one single BMU. The proposed rank-based BMU weighting scheme retains details without quantization or smoothing artifacts.

As the SOM learns from the raw data, and the weighted SOM results do not undergo further post-processing, our method is almost parameter-less, yet effective. We obtain a high-quality visualization that is helpful in several application scenarios. It is available within the open-source Gerbil HSI analysis framework at http://gerbil.sf.net.

Acknowledgement. We gratefully acknowledge funding of this work by the German Research Foundation (GRK 1773).

\section{References}

[1] J. Jordan and E. Angelopoulou, "Gerbil - A Novel Software Framework for Visualization and Analysis in the Multispectral Domain," VMV 2010: Vision, Modeling and Visualization, pp. 259-266, 2010.

[2] T. Kohonen, Self-organizing maps, vol. 30 of Springer series in information sciences, Springer, 3rd edition, 2001.

[3] J.S. Tyo, A. Konsolakis, D.I. Diersen, and R.C. Olsen, "Principal-components-based display strategy for spectral imagery," IEEE Trans. Geosc. Remote Sensing, vol. 41, no. 3, pp. 708-718, 2003.

[4] M. Cui, A. Razdan, J. Hu, and P. Wonka, "Interactive hyperspectral image visualization using convex optimization," IEEE 


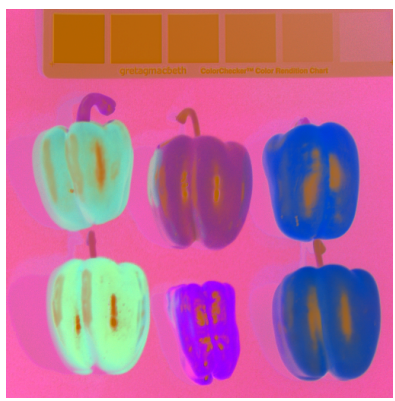

(a) PCA false-color visualization

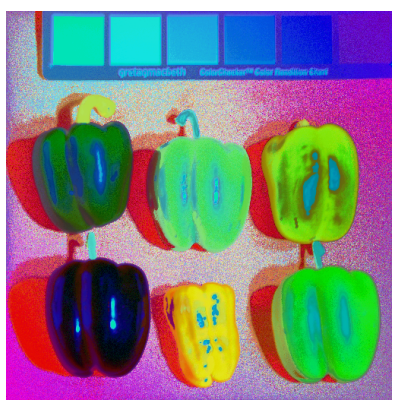

(b) SOM false-color visualization

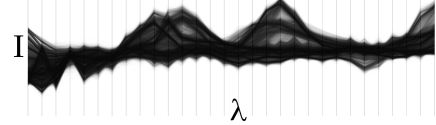

(c) Spectral gradient distribution

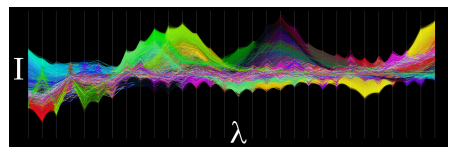

(d) Color-coded spectral gradient vectors

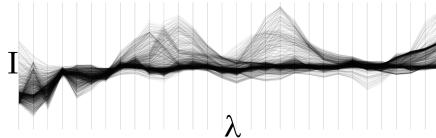

(e) SOM vector distribution

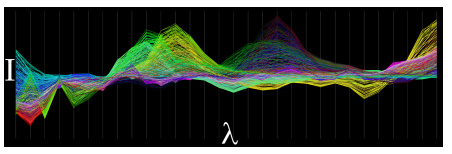

(f) Color-coded SOM vectors

Fig. 3. 31-band fake and real peppers image [15] depicting three organic and three plastic peppers. Left: Visualization results. Top right: Parallel coordinate plots of the image's spectral gradient distribution, and neuron vector distribution of the SOM trained on the image. Bottom right: Spectral gradient and SOM vectors colored in the same way as in (b). Parallel coordinate plots created with the Gerbil software [1].

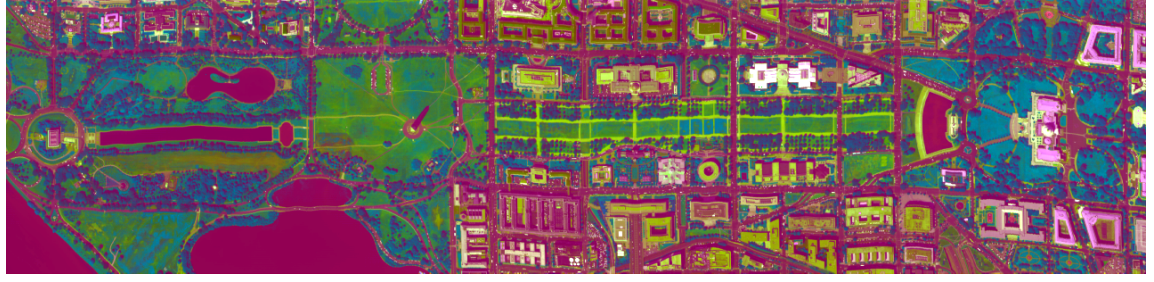

(a) PCA false-color visualization, $E_{\mathrm{R}}=0.535, E_{\mathrm{G}}=0.753, E_{\mathrm{B}}=0.761$

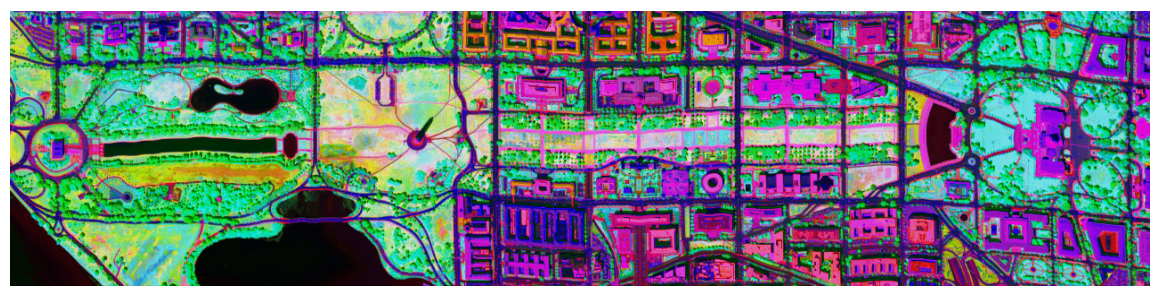

(b) SOM false-color visualization, $E_{\mathrm{R}}=0.957, E_{\mathrm{G}}=0.921, E_{\mathrm{B}}=0.932$

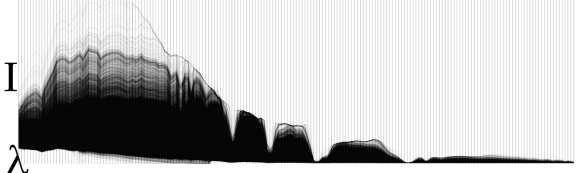

(c) Spectral distribution

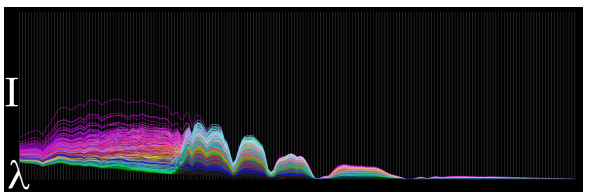

(d) Color-coded SOM vectors

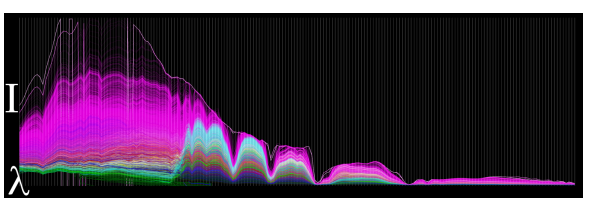

(e) Color-coded spectral vectors

Fig. 4. 191-band Washington D.C. Mall remote sensing image. Left: Visualization results. Top right: Parallel coordinate plot of the image's spectral distribution. Top bottom: Parallel coordinate plots of the neuron vectors of the SOM trained on the image, and the image's spectral vectors colored in the same way as in (b). Parallel coordinate plots created with the Gerbil software [1].

Trans. Geosc. Remote Sensing, vol. 47, no. 6, pp. 1673-1684, 2009.

[5] N.P. Jacobson and M.R. Gupta, "Design goals and solutions for display of hyperspectral images," IEEE Trans. Geosc. Remote Sensing, vol. 43, no. 11, pp. 2684-2692, 2005.

[6] J. Wang and C.-I Chang, "Independent component analysisbased dimensionality reduction with applications in hyperspectral image analysis," IEEE Trans. Geosc. Remote Sensing, vol. 44, no. 6, pp. 1586 - 1600, june 2006.

[7] C. M. Bachmann, T. L. Ainsworth, and R. A. Fusina, "Improved manifold coordinate representations of large-scale hyperspectral scenes," IEEE Trans. Geosc. Remote Sensing, vol. 44, no. 10, pp. 2786-2803, 2006.

[8] J.A. Lee and M. Verleysen, Nonlinear dimensionality reduction, Springer, 2007.

[9] T. Villmann, E. Merényi, and B. Hammer, "Neural maps in remote sensing image analysis," Neural Networks, vol. 16, no. 3, pp. 389-403, 2003.

[10] J. Vesanto, "Som-based data visualization methods," Intelligent data analysis, vol. 3, no. 2, pp. 111-126, 1999.

[11] J. Gorricha and V. Lobo, "Improvements on the visualization of clusters in geo-referenced data using self-organizing maps," Computers \& Geosciences, vol. 43, pp. 177 - 186, 2012.

[12] A. Manduca, "Multispectral image visualization with nonlinear projections," IEEE Trans. Image Processing, vol. 5, no. 10, pp. 1486 -1490, oct 1996.

[13] J. Fonville, C. Carter, L. Pizarro, R. Steven, A. Palmer, R. Griffiths, P. Lalor, J. Lindon, J. Nicholson, E. Holmes, and J. Bunch, "Hyperspectral visualization of mass spectrometry imaging data," Analytical Chemistry, vol. 85, no. 3, pp. 1415-1423, 2013.

[14] M. Sjöberg and J. Laaksonen, "Optimal combination of SOM search in best-matching units and map neighborhood," in $\mathrm{Ad}$ vances in Self-Organizing Maps, vol. 5629 of Lecture Notes in Computer Science, pp. 281-289. Springer, 2009.

[15] F. Yasuma, T. Mitsunaga, D. Iso, and S. K. Nayar, "Generalized Assorted Pixel Camera: Post-Capture Control of Resolution, Dynamic Range and Spectrum,” IEEE Trans. Image Processing, vol. 19, no. 9, pp. 2241-2253, Sept. 2010.

[16] Elli Angelopoulou, Sang W. Lee, and Ruzena Bajcsy, “Spectral gradient: a material descriptor invariant to geometry and incident illumination," in Computer Vision, 1999 Seventh IEEE International Conference on, 1999, vol. 2, pp. 861-867. 